\title{
An Energy Effective WBAN Architecture for Athletes Efforts Analysis under a Common Activity
}

\author{
Jyoti Kumari \\ Computer Science and Engineering, The NORTHCAP University, Gurgaon, 122017, India \\ E-mail: josahu98@gmail.com \\ Prachi \\ Computer Science and Engineering, The NORTHCAP University, Gurgaon, 122017, India \\ E-mail: prachiah1985@gmail.com
}

\begin{abstract}
Wireless Body Area Network (WBAN) is one of the most critical real time networks. In order to increase network lifetime, several protocols have been proposed in the Literature. Majority of these protocols are based on either coverage or residual energy of the network. However, none of the protocols is based on node criticality, communication count, coverage and residual energy. In this paper, we have taken into consideration all the above stated parameters to determine the optimal path for transmission of message. In particular, we are implementing this scenario for Athlete Activity Monitoring to identify the athlete with maximum physical stamina. As per best of our knowledge, only intra-BAN routing protocols are proposed till date. However, in this paper we have simulated Athlete Activity Monitoring scenario for intra-WBAN and interWBAN architecture. It is clear from the simulation results that our scheme performs much better in terms of energy consumption than earlier routing schemes.
\end{abstract}

Index Terms - WBAN (Wireless Body Area Network), Inter-BAN routing, Intra-BAN routing, Physical Strength, Communication Count, Node Criticality.

\section{INTRODUCTION}

WBAN is one of the advanced form of wireless networks that performs application specific communication. It is used in various fields like research, business, industrial, defense, and viable lifestyles. There are multiple small size sensors of limited energy in a WBAN. There are numerous applications of WBAN in different fields. One important application of WBAN is health care monitoring. The health of a person can be monitored by the analysis of physiological data collected by sensors placed on the patient body. Now a day's interest of users has increased in wearable wireless devices. Due to certain factors like topological changes, time-varying wireless channel, different channel bandwidths, low energy nodes etc., WBANs are not able to meet users interest level [7].
The design of WBAN includes layered architecture. Issues related with each layer are analyzed so that implementation, testing, debugging and enhance network management can be done effectively and efficiently.

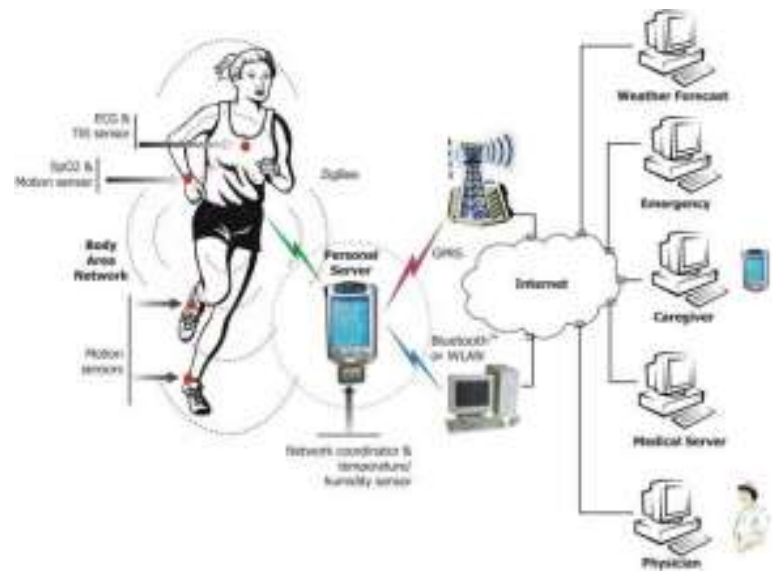

Fig.1. An Example of Wireless Body Area Network

A Wireless body area network is a static network with small no of nodes with energy specification. One of the application areas of WBAN is to monitor the health system of an athlete to identify the physical strength of the athlete. The presented work is focused on the same. Main motive of this paper is to study existing works in human physical movement recognition problem, and to propose and implement the work for multiple WBANs architecture which will remove the drawbacks of existing approaches.

In this presented work, multiple WBAN architecture is defined for some athletes with the placement of effective sensors to monitor physical strength of athlete under a common activity. One WBAN network is defined for each athlete. For this, Inter and Intra BAN communication is performed. The comparative measures on different BAN network are taken to identify the athlete having maximum physical stamina.

The remainder of this paper is organized as follows: Existing works are discussed in Section II. In section III, 
proposed work is presented. Section IV presents the network design of the presented work. Comparative analysis of existing routing approach and the proposed algorithm is presented in the section V. Finally, in section VI paper is concluded with future scope.

\section{LITERATURE SURVEY}

Lots of works is already done by different researchers for body area network to optimize the capabilities under different aspects. These aspects include the architectural improvement, localization improvement, routing approaches etc. Some of such work is defined specific to the application area. Most of the work done by the researchers is on health monitoring systems $[8,9]$ for the patients. Some work is also done in different application areas such as soldiers monitoring [10], war area analysis, chemical plant worker analysis, athlete analysis[11,12] etc. Some of the researchers presented the network models and algorithms to optimize the network strength, communication and effectiveness. Some of such work defined by earlier researchers is given in this section.

Kihyun Kim [1] has defined a work on effective routing in postural analysis in the body area network. The effective communication is achieved in stationary sensor network for human body network. The safer deployment of nodes was done under coverage hole identification. The coverage perspective analysis was defined under body movement analysis so that the effective communication with network nodes can be performed. Solution to the network problem, including the mobility analysis, fault tolerance and scalability of network was provided. The protocol level work was defined to achieve the communication under energy effective scenario and routing scheme under the global positioning system. The author improved the energy level formation of nodes over the network.

SEA-BAN (Semi-Autonomous Adaptive Routing in Wireless Body Area Networks) [2] was proposed which combined the advantages of both single hop transmission and multi hop transmission. In this algorithm, data of all nodes are transmitted to the BNC (Body Node coordinator) which performs computations on this data. This leads to reduced computational burden on sensor nodes. All sensor nodes are assumed to be within coverage range of $\mathrm{BNC}$. This algorithm significantly improved the maximum network lifetime as compared to existing direct and multi hop transmission models.

In [3], the author proposed an algorithm EAR-BAN (Energy Efficient Adaptive Routing in Wireless Body Area Networks) which is an extension and modification of SEA-BAN algorithm. EAR-BAN has considered the coverage distance along with remaining energy while performing routing. It is clear from the simulation results that EAR-BAN has successfully enhanced the network working lifetime better than other existing WBAN routing protocols. But there exists still some issues like BNC position, node criticality, communication count, body tissue heating etc. which need to be resolved.

Arash Maskooki [4] has defined a work on opportunistic routing for body area network. The interaction of network with outer world is defined along with energy preserving communication over the network. The link estimation performed to provide the significant network improvement. The author analyzed the quality of network and the links under frequently changing network so that effective WBAN communication can occur. The analysis on problem domain was done to generate the opportunistic scheme based on body movement analysis. To optimize routing, the life time based comparison was done for route generation in effective time.

Samaneh Movassaghi [5] has defined an energy adaptive approach for power aware routing in body area network. The author defined deployment of nodes for the body area network. The author provided the energy adaptive communication in body area network. To provide the reliable and effective communication in the body area network. The author provided the optimization to the system so that the reliable and effective network transformation will be achieved. The author provided the analysis on routing protocol to improve the network communication and improve the network life.

N. Javaid [6] defined a work to measure the fatigue of soldiers in the body area network. A routing protocol was proposed for measuring the fatigue of a soldier. To perform monitoring of specific attributes, three sensors were placed on the soldier's body. The author proposed an event driven protocol which considers three cases for fatigue measurement of a soldier. The parameters used for the work evaluation were network lifetime, throughput, remaining energy of sensors and the fatigue of a soldier.

It is found that all existing works are performed for single BAN network. Work on multiple BAN networks is yet to be performed. The routing approaches have considered remaining energy and coverage distance parameters for routing. The proposed work is focused on energy efficient routing in multiple BAN networks by considering remaining energy, coverage distance parameters, node criticality and communication count parameters.

\section{PROPOSED WORK}

In the existing works [2, 3], an energy effective cluster specific multi-hop routing approach is defined to perform the communication in WBAN network. The route selection parameters included are available energy and the distance vector. This model is based on the priority level formation of nodes so that the default communication to the network will be defined. This existing model includes nodes synchronization approach to control the network communication under energy parameters. The author has used the algorithmic framework to represent each stage of work.

In the proposed work the author has considered node criticality and communication count parameters along with the earlier available energy and distance vector parameters for routing. Inclusion of these parameters has significantly improved the network lifetime and reduced 
communication energy wastage.

In this work, the analysis on the multiple athlete's health will be performed. It means, the work will be performed on integrated multiple BAN architecture. Each BAN architecture will be defined for each athlete and the communication analysis will be performed for each athlete. The work is defined here to handle the multipleBAN architecture along with their inter-communication and intra-communication analysis. The objective of work is to identify the most effective BAN architecture to analyze the physical strength of athlete. Higher the efforts made by an athlete, more energy consumption will occur in BAN network. The presented work includes analysis of the multiple BAN architecture under particular common activity to identify the athlete with maximum physical stamina. The analysis of the work will be performed in terms of communication analysis, network life analysis and athlete efforts analysis.

Table 1. Flow of Work

1. Set up the BAN architecture with the physical placement of sensors to monitor different activities. The setup will be performed with respective to the athlete.

2. Perform the communication over the network based on the activity or the performance measures of the individuals.

3. Perform the intra BAN communication to analyze the athlete's activity.

4. Generate the overall performance measure in terms of energy consumption and network life.

5. Perform the inter-BAN communication between different BAN architectures if required.

6. Perform the comparative measures between these BAN architectures to monitor the individual network performance.

7. Perform the analysis of work in terms of athlete effort analysis and network communication analysis.

8. Identify the high performance athlete under these measures.

The network processing is done here under localization of network along with architectural specifications. Once the network is established, network communication is performed under sensing range and load vectors. The routing communication control over the network is provided. The work is defined under energy and network optimization so that reliable and effective network route over the network will be established.

\section{Proposed Routing Algorithm For Intra BAN Routing}

The routing within the single BAN network is defined by the specification of the source and the destination node over the network and to perform the communication so that the reliable communication route over the network will be established. The algorithm for the route generation over the body area network is given hereunder.

\section{Algorithm(N, Src, Dst)}

/* Define a Network with $\mathrm{N}$ Nodes with Src and Destination*/
1. For $\mathrm{i}=1$ to $\mathrm{N}$

[Define $\mathrm{N}$ nodes with the Position and Energy Specification]

\{

Nodes (i). Position=Random

Nodes (i). Type $=$ Random $(1,3)$

Nodes (i). Energy=Random

Nodes(i). CommCount $=0$;

\}

2. Set CurNode $=$ Src

[Set Source Node as Current Node]

3. While CurNode != Dst

[Repeat Process Till Destination Node not Occur] \{

Generate the Neighbor List for CurNode called NeighList

4. Set nexthop=NeighList (1)

5. For $\mathrm{i}=1$ to NeighList. Length

6. If (Nodes(i).Energy > EThreshold And Distance(i, Dest $)<$ Distance $($ CurNode, Dest $)$ )

7. \{

8. If (NeighList(i) . Energy > Nodes(nexthop).Energy And NeighList(i).Type $=$ Info And NeighList(i).CommCount < Threshold And NeighList (i).Distance $<$ SensingRange )

9. Set nexthop $=\mathrm{i}$;

\}

10. Else If (NeighList(i).Energy > Nodes(nexthop). Energy And NeighList(i).Type = Data And NeighList (i).Distance < Sensing Range)

\{

11. Set nexthop $=\mathrm{i}$;

\}

12. Else If (NeighList(i).CommCount < Threshold And NeighList(i).Type $=$ Data And NeighList(i).Distance $<$ Sensing Range)

\{

13. Set nexthop $=\mathrm{i}$;

\}

\}

\}

14. Nodes(nexthop).Energy = Nodes(nexthop).EnergyForwarding Energy;

15. Path. Add (next hop)

16. Nodes(nexthop).CommCount $=$ Nodes(nexthop). CommCount+1

17. Set CurNode $=$ nexthop

\}

\}

18. $\operatorname{Nodes}(\operatorname{Src})$.Energy = Nodes(Src).Energy Transmission Energy;

19. Nodes(Dst).Energy = Nodes(Dst).Energy Receiving Energy;

This algorithm has considered the communication count and node criticality along with the remaining energy and the coverage distance parameters for routing in WBAN. These two additional parameters will help in 
achieving maximum network lifetime and will minimize energy consumption in WBAN.

\section{NETWORK DESIGN}

In this section, the network architecture specification for the multiple BAN architecture is presented for the generation of network area in the critical application specification. In this work, the example of this architecture for the athlete analysis network is used.

Here proposed work is implemented for $6 \mathrm{WBAN}$ networks. In each network there are 7 sensor nodes attached on different body parts. Each node except the controller, collect data about one physiological parameter like body temperature, glucose level, pulse rate, etc. The controller node collects data of remaining nodes and transmit it to the network coordinator. Network coordinator after receiving data of all 6 networks, determine athlete with highest physical stamina by comparing the energy consumption values of all the networks. The network with lower remaining energy value has highest higher energy consumption. Hence network with lowest remaining energy value will have athlete with highest physical stamina.

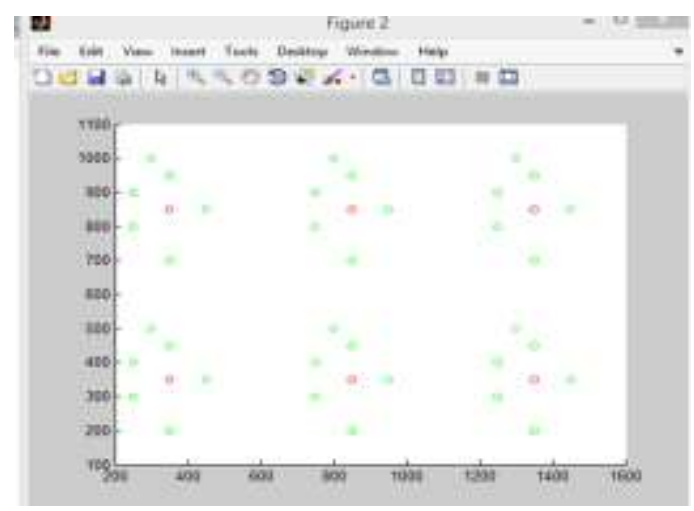

Fig.2. Multiple WBAN Architecture

In fig 2, network design is shown. Controller node is represented by green color circle and red color circle represent sensing nodes.

\section{Network Scenario}

Here the network is defined to control the network communication in multiple BAN networks defined under the application specification. The parameters of the network are given hereunder.

Table 2. Network Scenario

\begin{tabular}{|l|l|}
\hline Parameters & Values \\
\hline Number of Nodes & 7 \\
\hline Initial Energy & $1 \mathrm{~J}$ \\
\hline Transmission Energy & $50 \mathrm{n} \mathrm{J}$ \\
\hline Forwarding Energy & $10 \mathrm{n} \mathrm{J}$ \\
\hline Receiving Energy & $30 \mathrm{n} \mathrm{J}$ \\
\hline Type of Data & Normal and Video \\
\hline Packet Size (Normal) & $4 \mathrm{~K}$ \\
\hline Packet Size (Video) & $32 \mathrm{~K}$ \\
\hline
\end{tabular}

\section{RESULTS}

In this section, the comparative results of existing routing approach and proposed routing approach are presented. The comparison over the network is here presented in terms of network communication and network life parameters. Tool used for experimental study is Mat lab.

This section evaluates the performance of existing routing approach with the proposed routing approach. Here energy level comparative results are shown over the network. Round means network operation time in which nodes send data to controller node. We assume that duration of a round is one second. The proposed algorithm is expressed as proposed in the graphs and the previous algorithm represented as existing.

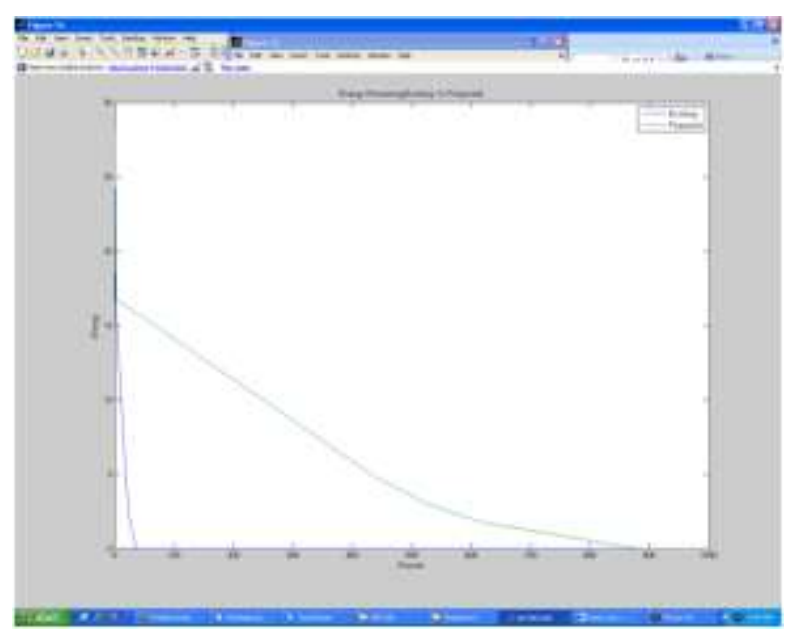

Fig.3. Energy Level Analysis (Existing v/s Proposed)

Here energy level comparative results are shown over the network. Here the $\mathrm{x}$ axis represents the communicating rounds and the $\mathrm{y}$ axis represents the network energy. The figure shows that the energy level for the network is about $30 \mathrm{~J}$ for both existing and proposed network. But the existing approach has given the higher energy loss. In existing work, the network lost all its energy after 50 rounds whereas in case of proposed work, the network energy resides for 1000 rounds.

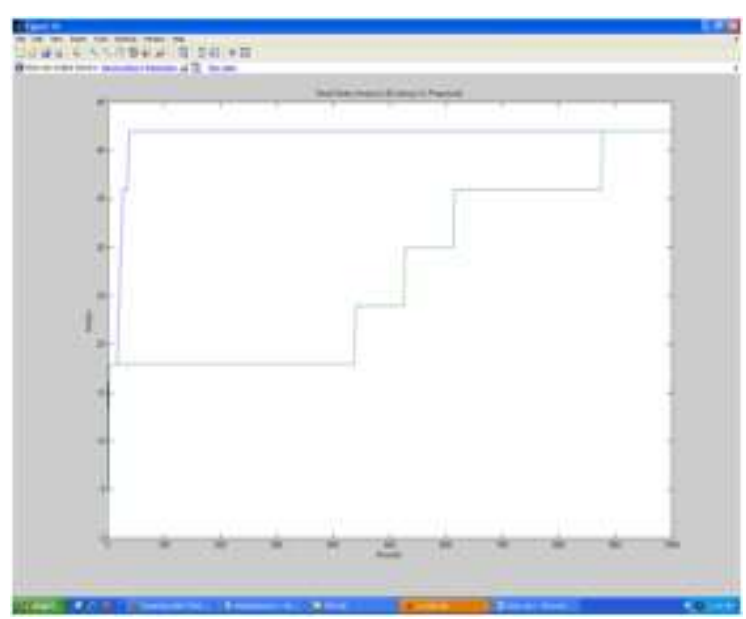

Fig.4. Dead Node Analysis (Existing v/s Proposed) 
Here fig 4 is showing the analysis of existing and proposed approach in terms of network life. The network life is here identified in terms of alive nodes. The figure shows that all nodes are alive initially but after 50 rounds no node is alive in existing approach whereas in case of proposed work, the no node is alive after 900 rounds.

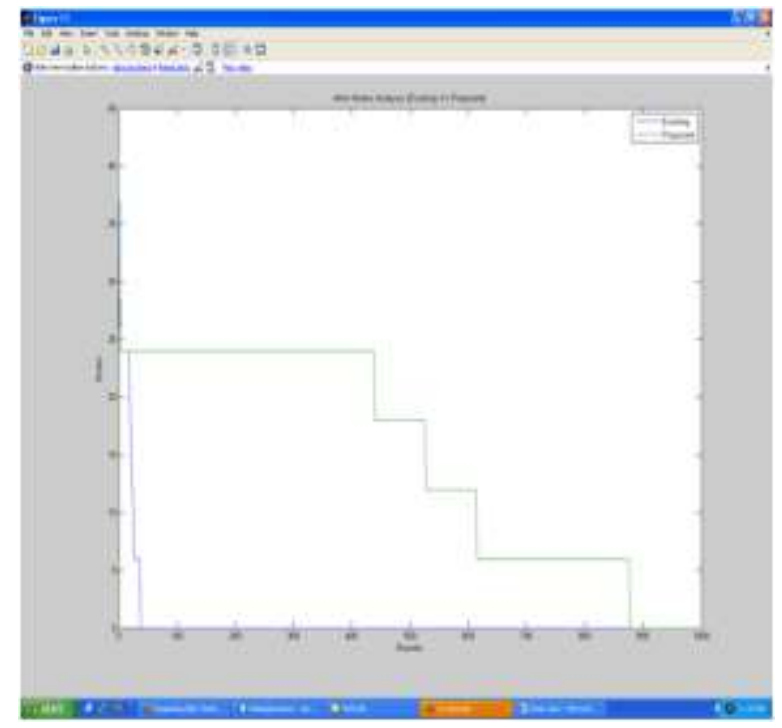

Fig.5. Alive Node Analysis (Existing v/s Proposed)

Here fig 5 is showing the analysis of existing and proposed approach in terms of network life. The network life is here identified in terms of alive nodes. The figure shows that all nodes are alive initially but after 50 rounds no node is alive in existing approach whereas in case of proposed work, the no node is alive after 900 rounds.

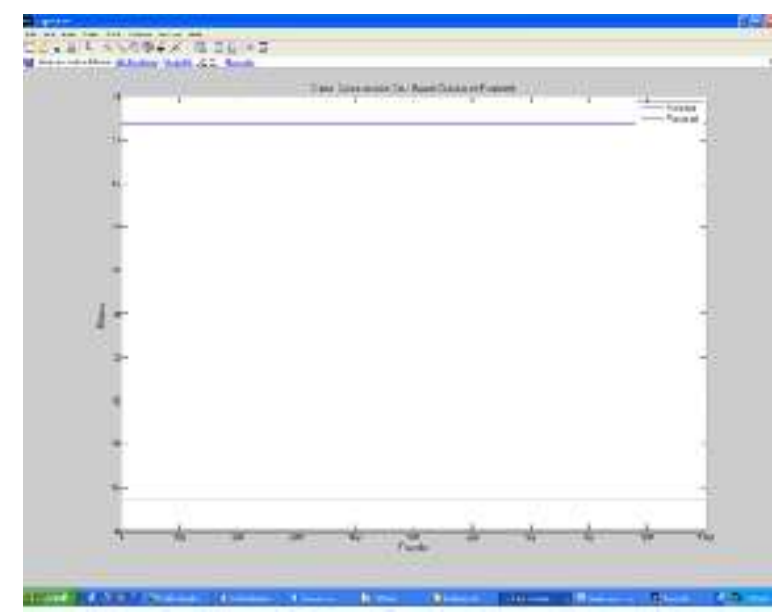

Fig.6. Aggregative Energy Analysis

Here the aggregative energy analysis over the network nodes is presented. Here $\mathrm{x}$ axis represents the communication rounds and the $y$ axis represents the aggregative network energy. Here energy effective communication over the network is performed. The aggregative energy loss in existing approach is about 23J whereas in case of proposed approach it is about 5.5J. The results show that the proposed work has reduced the energy consumption over the network.

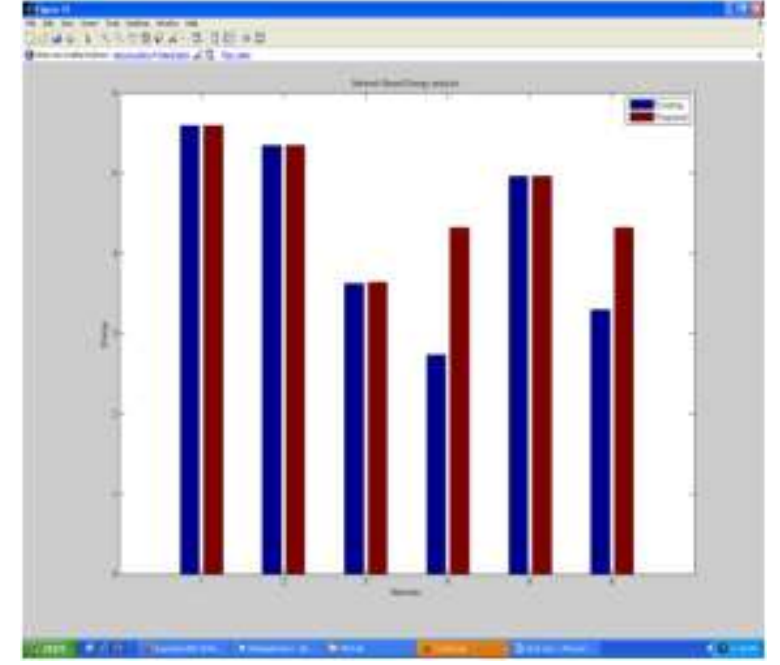

Fig.7. Energy Consumption Level Analysis

Fig: 7 is here defined to perform network energy analysis. Here each bar represents the energy analysis on each player of the network. The figure shows that the energy consumption in most of the networks is same. But where the path establishment is critical, the proposed work is effective. The energy level of network 4 and network 6 is higher in case of proposed work.

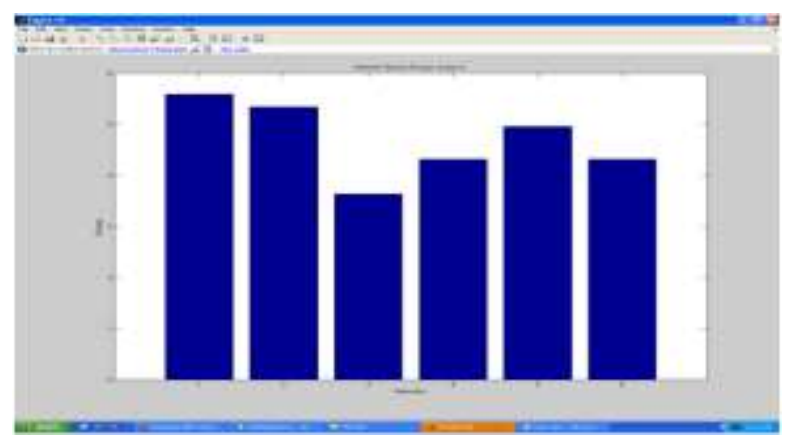

Fig.8. Energy Consumption Analysis

In fig 8 network energy analysis is performed. Here each bar represents the energy analysis on each player of the network. The figure shows that the network 1 is having the highest energy where as the least energy is on network 3 .

The results are implemented in Mat lab environment. The comparative results show that the presented work has improved the network communication and network life. The results shows that in case of existing work, the network energy remains only up to 50 rounds whereas in case of proposed work, the network life retains up to 900 rounds. It shows the significant improvement in the network life From fig5, it is clear that the most of the networks are having the equal energy consumption and life for existing and proposed approach. But for critical networks 4 and 6 , the significant improvement is identified in terms of network life and energy remaining in the network.

When the individual networks in proposed work are compared, it is found that the energy remaining in $1^{\text {st }}$ network is higher so that the BAN 1 has proven its 
strength in terms of its long lasting capability and athlete stamina is weakest. In same way, the energy remaining for BAN 3 is least which shows the athlete with highest stamina.

\section{CONCLUSION AND FUTURE WORK}

In this work, an effective model is defined in a communication control mechanism for multiple BAN networks. The work is defined here to process the network architecture for specialized applications such as monitoring the efforts of athletes or players during some activity or the game. A routing algorithm is proposed and implemented to transmit data between the source and the destination. This algorithm has considered the four parameters which were not adopted earlier by any existing routing approach. The parameters are remaining energy, coverage distance, node criticality and communication count. Either the coverage distance or remaining energy was considered as routing parameter by existing routing approach. The proposed work including all four parameters resulted into higher network lifetime and less energy wastage.

The presented work is defined in two main phases. In the first phase, the communication in the individual body area network is being analyzed and presented with a specification of network route constraint. In this work, an effective communication route is generated over the single body area network under multiple analytic vectors. In the second phase, the comparative analysis between different Body Area Networks is done. The results are implemented in Mat lab environment. The comparative results show that the presented work has improved the network communication and network life. The results shows that in case of existing work, the network energy remains only up to 50 rounds whereas in case of proposed work, the network life retains up to 900 rounds. It shows the significant improvement in the network life. The analysis is also obtained in different networks. The results shows that the energy remaining for different networks and life of different network in case of proposed work is higher.

The proposed work is implemented with 6 BAN networks for 6 athletes. It is found that BAN 3 has minimum remaining energy and highest energy consumption. This means higher efforts leading to higher physical stamina. So, BAN 3 athlete has highest stamina. Likewise, BAN 1 has highest remaining energy which means BAN 3 athlete has lowest stamina.

The comparative analysis is performed under decision vectors such as energy, network life and communication. In future some criticality parameters can also be used for analysis. In this work, the routing is considered as the main operation to analyze the communication behavior over the network. In future some other communication aspects over the network can be considered for analysis.

\section{REFERENCES}

[1] Kihyun Kim, "An Efficient Routing Protocol based on Position Information in Mobile Wireless Body Area Sensor
Networks", 2009 First International Conference on Networks \& Communications 978-0-7695-3924-9/09 () 2009 IEEE.

[2] Md. Tanvir ul Huque et al., "SEA-BAN: Semi Autonomous Adaptive Routing in Wireless Body Area Networks", 7th International Conference on Signal Processing and Communication Systems, IEEE 2013, pp. 1-7

[3] Md. Tanvir ul Huque et al., "EAR-BAN: Energy Efficient Adaptive Routing in Wireless Body Area Networks", 7th International Conference on Signal Processing and Communication Systems, IEEE 2013, pp. 1-10.

[4] Arash Maskooki, "Opportunistic Routing for Body Area Network", 1st IEEE International Workshop on Consumer eHealth Platforms, Services and Applications 978-1-42448790-5/11@2011 IEEE.

[5] Shruti P. Mahambre, "Decentralized Adaptive Routing for Reliability in Event Broker Networks", 2009 15th International Conference on Parallel and Distributed Systems 1521-9097/09 () 2009 IEEE.

[6] N. Javaid, "Measuring Fatigue of Soldiers in Wireless Body Area Sensor Networks", 2013 Eighth International Conference on Broadband, Wireless Computing, Communication and Applications 978-0-7695-5093-0/13 (C) 2013 IEEE.

[7] Samaneh Movassaghi, "Wireless Body Area Networks: A Survey", IEEE Communications Surveys \& Tutorials, 2013 IEEE, volume 16, pp. 1658-1686.

[8] Aravind Kailas, "Wireless Communications Technology in Telehealth Systems", 1st International Conference on Wireless Communication, Vehicular Technology, Information Theory and Aerospace \& Electronics Systems Technology, VITAE 2009 IEEE, pp. 926-930.

[9] Xiaoling Wu, "Optimal Routing in Sensor Networks for Inhome Health Monitoring with Multi-factor Considerations", Sixth Annual IEEE International Conference on Pervasive Computing and Communications 0-7695-3113-X/08 2008 IEEE.

[10] N. Javaid, "Measuring Fatigue of Soldiers in Wireless Body Area Sensor Networks", 8th International Conference on Broadband, Wireless Computing, Communication and Applications, 2013 IEEE, pp. 227-231.

[11] Ashay Dhamdhere.et.al, " Experiments with Wireless Sensor Networks for Real Time Health Monitoring", 35th Conference on Local Area Networks, IEEE 2010, pp. 938945.

[12] Zongjian He .et.al, "A Wearable Wireless Body Area Network for Human Activity Recognition", 6th International Conference on Ubiquitous and Future Networks, 2014 IEEE, pp. 115-119.

\section{Authors' Profiles}

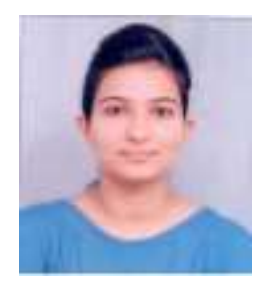

Jyoti Kumari is M.Tech holder in Computer Science from ITM University of Gurgaon, India. Her current research interests include wireless Body Area Networks.

She received the B.Tech degree from B.P.S Women University Khanpurkalan of Haryana, India in 2013.

She is author of article "A Comprehensive Survey of Routing Protocols in Wireless Sensor Networks" IEEE indexed conference. 


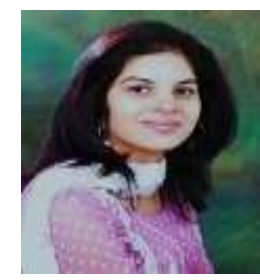

Prachi is Ph.D. holder in Computer Science from the Banasthali University of Rajasthan, India. Her current research interests include wireless sensor networks, key agreement in wireless peer-to-peer systems and security in underwater sensor networks. Prachi received the B.Tech. degree from M.D. University, Rohtak in 2007 and the M.Tech. degree with distinction in Computer Science from the Banasthali University at Rajasthan in 2009. She is author of 21 refereed articles in these areas, 9 in peer reviewed, reputed international journal indexed in international databases and 12 in IEEE and Springer indexed International Conferences.

How to cite this paper: Jyoti Kumari, Prachi,"An Energy Effective WBAN Architecture for Athletes Efforts Analysis under a Common Activity", International Journal of Information Technology and Computer Science(IJITCS), vol.7, no.11, pp.74-80, 2015. DOI: 10.5815/ijitcs.2015.11.09 